\title{
Active repurposing of drug candidates for melanoma based on GWAS, PheWAS and a wide range of omics data
}

\author{
Ali Khosravi ${ }^{1}$, B. Jayaram², Bahram Goliaei ${ }^{3}$ and Ali Masoudi-Nejad ${ }^{1 *}$
}

\begin{abstract}
Background: Drug repurposing is a swift, safe, and cheap drug discovery method. Melanoma disorders present low survival and high mortality rates and are challenging to diagnose and treat. Moreover, there is a high volume of worldwide investigations that are attempting to find melanoma-related genes of influence, which can be identified as responsive targets for reliable treatment.
\end{abstract}

Method: In this study, we used a wide range of data analyses to analyze over 1100 genes and proteins of influence with respect to cutaneous malignant melanoma. Our analysis included various investigational results from genomeand phenome-wide association studies (GWAS and PheWAS, respectively), biomedical, transcriptomic, and metabolomic datasets. We then researched the DrugBank for potential melanoma targets from the selected list. We excluded known melanoma targets to obtain a list of druggable proteins. We performed a precise analysis of the drugs' pathogenesis and checked the expression profiles of the selected drugs having high associations with known anti-melanoma drugs.

Result: We found 35 drugs that interacted with 20 unique targets. These drugs appear to have high melanoma treatment potentials. We confirmed our results with previous studies and found supporting references for 30 of these drugs. In conclusion, this investigation can be applied to various diseases for the efficient and economical repurposing of various drug compounds. For further validation, the results may be applicable for in vivo tests and clinical trials.

Keywords: Drug repurposing, Melanoma, GWAS, PheWAS, Transcriptomic, Metabolomics, Medical dermatology, Oncology, Drug response

\section{Background}

Cutaneous malignant melanoma is one of the most perilous diseases in the world, affecting more than millions of people globally. According to world health organization, there are over 2 to 3 million cutaneous malignant melanoma diagnoses every year. Recent reports from the American Cancer Society revealed that the five-year survival rate for patients with early stage detection of skin cancer is approximately $99 \%$ in the U.S. Cutaneous malignant melanoma has excessive morbidity and mortality rates (Cummins et al. 2006). The survival time can drop to $63 \%$ when the disease affects lymph nodes, and it can lower to $20 \%$ in regard to metastasize other organs (Balch et al. 2009).

\footnotetext{
* Correspondence: amasoudin@ut.ac.ir; http://LBB.ut.ac.ir

${ }^{1}$ Laboratory of Systems Biology and Bioinformatics (LBB), Institute of

Biochemistry and Biophysics, University of Tehran, Tehran, Iran

Full list of author information is available at the end of the article
}

Disease etiology is the most important step towards finding a feasible treatment for any disease. According to reports that were published earlier, melanoma is mainly due to the influence of the UV-damage response to genomic content in humans. Genomic instability caused by UV-radiation and other factors have various resources (Parkin et al. 2011), including the influence of the tumor suppressor gene, cell cycle inhibitors, oncogene activities, molecular and cytogenetic changes and telomere dysfunction, and others (Elder 1999). Although these resources are mainly induced by internal genomic content, according to the genetic interpretation of the population, environmental factors are key operating agents for such diseases (Han et al. 2005).

Understanding the disease etiology can highlight the inducing genes, which are directly or indirectly affected by the progress of cancer. In this study, we collected

(c) The Author(s). 2019 Open Access This article is distributed under the terms of the Creative Commons Attribution 4.0 International License (http://creativecommons.org/licenses/by/4.0/), which permits unrestricted use, distribution, and 
influencing gene from multiple sources, with strong biological evidence. Genome-wide association studies (GWAS) (MacArthur et al. 2017), phenome-wide association studies (PheWAS) (Denny et al. 2013), transcriptome analysis (Berger et al. 2010), metabolomics analysis (Wishart et al. 2018a) and disease-gene association studies (Babbi et al. 2017) are used to reveal these associated genes.

Apart from finding the genes that are associated with melanoma, it is highly important to find a therapeutic solution to reduce the number of patients who are suffering. Drug repurposing (or drug repositioning), which has become a conventional drug development procedure, can reuse already available, well-studied, marketed drugs for new indications (Li et al. 2016). Drug repurposing can reduce the time for identification of the leading compound for 3-12 years; it can also reduce the risk and lower the cost of drug development (Ashburn and Thor 2004). In this study, we used the "Disease Based Approach" as one of multiple drug-repurposing approaches (Sun et al. 2017; Zhang et al. 2015; Li et al. 2016). Hence, the leading compound will be identified by an analysis of target-based interactions with the associated genes corresponding to a well-known disease.

In this study, systematic approaches are involved in finding drug compounds for melanoma treatment. We have approached advanced biological technology, such as GWAS, PheWAS, expression profiles, and biomedical and metabolomics data, to obtain the highest operational druggable biomarker and their corresponding leading molecule from already available drugs. We finally used the text-mining approach to validate our candidate drug compound from previous studies.

\section{Materials and methods Dataset collection}

To obtain the dataset for skin cancer-related genes, proteins and metabolites, we used several databases and catalogs. In this study, we used genomics, transcriptomics and metabolomics, and biomedical data from various sources, which is described as follows.

\section{Genome wide association studies}

We have revealed all associated genes for melanoma disorders from the GWAS catalog (MacArthur et al. 2017). We have collected information on 1) associated genes; the nearest gene associated with an allele; 2) SNPs, the unique SNP number; 3) patient ethnicity, the ethnicity of population study; 4) publication information, PubMed ID; 5) $P$-value, the $p$-value of the allelic association to the disorder; and 6) the phenotypic trait from the catalog; associated disorder. The selection of the disease/trait attribute as "melanoma" and "cutaneous malignant melanoma" has been done in this process.

\section{Phenome wide association studies}

Utilization of phenome-wide association studies (PheWAS) for drug repurposing has been done successfully during past years (Rastegar-Mojarad et al. 2015; Yin et al. 2018; Khosravi et al. 2019). In this study, we have used the PheWAS catalog to discover all associated genes corresponding to variant alleles for different ethnicities around the world (Denny et al. 2013). The comorbidity of different phenotypic traits has been traced by variation in allelic content due to a shared biological mechanism and/or environmental effect, such as UV radiations (Bush et al. 2016). We have retrieved the information from the catalog by setting the phenotype as "melanoma" and "skin cancer" and extracted the following data: 1) SNPs, SNP accession number; 2) PheWAS phenotype, the associated disorder; 3) $p$-value, the p-value corresponding to the association of SNPs and disorders; and 4) gene name; nearest gene associated with an allele.

\section{Metabolomics data}

Metabolomics analysis is used significantly as a drug discovery methodology. The analysis highlights preclinical research and biomarker detection. Cancer-related metabolites are associated with enzymes and transporters as the melanoma biomarkers are leading targets for a drug-repurposing methodology (Robertson and Frevert 2013). It can be used as patient stratification and help translational medicine discovery (Srivastava and Creek 2018). In this study, we have used the Human Metabolome Database (HMDB) (Wishart et al. 2018a) for mining melanoma-related proteins. We retrieved the information by exploring all the metabolites that are associated with melanoma disorder by searching "melanoma" and "malignant melanoma" from the database. In next step, we manually collected the proteins that are associated with the respected metabolites.

\section{Transcriptome data}

RNA-Seq and transcriptomics analysis can improve the productivity of biomedical research to obtain more precise compounds in the drug discovery process (Zhao et al. 2014). As genomic mutation and their corresponding epigenetic changes can alter gene expression and functions, the study of DNA microarrays has great importance (Atak et al. 2013). Misplacement in the DNA copy number can be dignified by the DNA microarray and can also alter gene expression profiles (Kumar-Sinha et al. 2015). The collected dataset was in terms of differentially co-expressed genes that are affected by melanoma disorder (Berger et al. 2010). The study provided differentially co-expressed gene names as leading targets for the identification of anti-melanoma drug compounds. 


\section{Biomedical data}

A modern biomedical technique has enhanced genetic research by discovering different genetic component of phenotypic traits. We have used eDGAR database (Babbi et al. 2017) to reveal skin cancer-associated genes with annotated relationships among them. eDGAR collected disease-based associated genes deposited in OMIM (Amberger et al. 2015), UniProt (UniProt Consortium 2015) and CLINVAR (Landrum et al. 2016) databases. These genes were revealed from the phenotype OMIM ID 155600 corresponding to cutaneous malignant melanoma.

\section{Metabolites protein network reconstruction}

We reconstructed the metabolites enzymes/transporters networks by mapping their interconnections derived from metabolomics data. We prepared a flat-file, which states metabolites and their associated protein and the type to reconstruct the network. As you can see in the result section 3.2, we visualized the network with the help of Cytoscape tools (Shannon et al. 2003).

\section{Drug mapping}

We collected all melanoma-associated genes and proteins from GWAS, PheWAS, transcriptomics, metabolomics and biomedical studies as described above. The method has been shown in Fig. 1. This set contains more than 1100 associated genes and proteins having the maximum influence on the disorder. You can find the dataset in supplementary file 1 (https://github.com/ LBBSoft/Melanoma). We tracked different drug-related databases and found DrugBank 5.0 (Wishart et al. $2018 \mathrm{~b})$ to be one of the best updated databases containing approximately 17,000 drug-target associations and information on over 10,000 drug compounds. We have mapped the drug-target information with our set of genes and proteins in the DrugBank database. We have revealed the drug name, mode of action, target name, current indications and drug groups from the DrugBank. We also excluded all experimental, illicit, withdrawn and investigational molecules from our data and only considered the approved drug-target information in this analysis. We have excluded 638 unique drugs interacting with 165 target genes, which are available in supplementary file 2 (https://github.com/LBBSoft/Melanoma).

\section{Pathogenesis information validation}

We have analyzed the pathogenic nature of the revealed drug molecules to comprehend drug antagonists or agonists (Wang and Zhang 2013). We revealed antimelanoma pathogenesis information through biomedical records and literature reviews from PubMed central. We analyzed the dataset for pathogenetic information of genes, such as the gain of function (GOF) and loss of function (LOF) roles in humans for anti-melanoma efficacy. We used the OMIM (Amberger et al. 2015) database mainly to obtain this information. As you can see in the results section 3.5, we removed the genes/proteins with suitable pathogenetic information with respect to the transporter action of the drug and maintained genes/proteins with reliable scientific reports.

\section{Connectivity map analysis}

The connectivity map (CMap) is a drug-response expression profile analysis on various human-cultured cell lines. This approach shows a transcriptional expression profile on the treatment of drugs on humancultured cell lines (Lamb et al. 2006). CMap qualifies the drug target associations that are correlated to melanoma through gene expression profiles. We have analyzed all drug candidates from the above states in CMap to comprehend their mechanisms of action and anti-cancer effects.

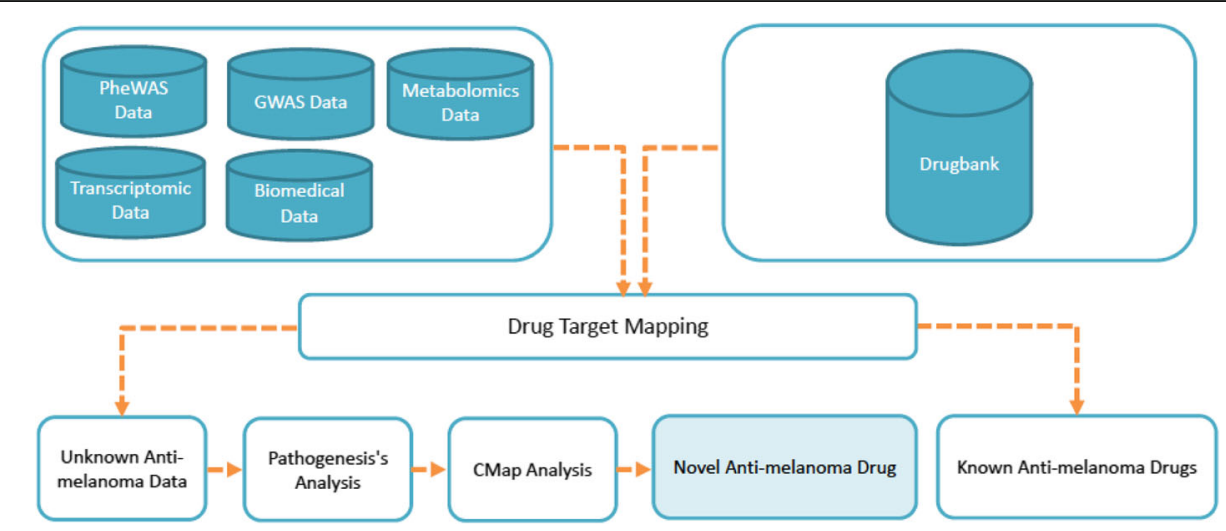

Fig. 1 Drug repurposing approach to identify novel anti-melanoma drugs; in this approach we have collected melanoma responsive genes/proteins and identified target genes by drug target mapping with the help of drugbank. We have removed all known anti-melanoma drugs and analyzed the unknown anti-melanoma drugs by pathogenesis information and CMap analysis. We have found 35 drugs interacting with 20 targets 


\section{Results}

\section{Systematic collection of melanoma-related biomarkers}

As described above, the melanoma-related GWAS dataset consists of 55 unique genes mapped by different SNP-risk alleles. The minimum and maximum $p$-value of collected data are 4E-37 and 8E-06, which are used for high demand and an accurate analysis. The $P$-value in GWAS and PheWAS studies is a probability of the type I error that is made in hypothesis testing. The Pvalue signifies the possibility of randomness in finding any disease associated to a specific variant. These data have been compiled from 9 different studies. Similarly, we have revealed 765 alleles by looking for "melanoma and skin cancer" keywords in the PheWAS catalog. These SNPs correspond to 260 unique associated genes in our analysis. The $p$-value of the revealed genes is in the range of 0.05 to $1.106 \mathrm{E}-16$.

We exposed 23 metabolites that are associated with 616 unique proteins in the metabolomics dataset with significant actions in the form of enzymes, transporters and unknowns in melanoma. In addition, we included 27 validated differentially expressed genes from the melanoma transcriptome. Finally, we incorporated 11 unique genes that are associated with cutaneous malignant melanoma from biomedical databases. The set of genes and proteins has 1178 unique members. These members have been identified and were discovered from different approaches, and some of them have been confirmed from more than one approach. The distribution of genes is available in Fig. 2.

\section{Metabolites protein network analysis}

We found approximately 800 metabolite-protein associations related to melanoma disorders in the dataset. The proteins in the dataset were in the form of enzymes, transporters or sometimes unknown. To rely on the dependability of our finding, we reconstructed a protein-metabolite network consisting of proteins/metabolites as nodes and their associations as edges. This highly connected graph, which is shown in Fig. 3, was visualized by Cytoscape (Shannon et al. 2003). There are 23 unique metabolites connecting to 617 associated enzymes or transporters.

\section{Drug-target mapping}

All melanoma risk biomarkers, which consist of a set of genes and proteins corresponding to melanoma disorder, are gathered here. We have four types of targets in the form of protein (215 drugs associated with 127 targets), enzyme (604 drugs associated with 56 targets), transporter

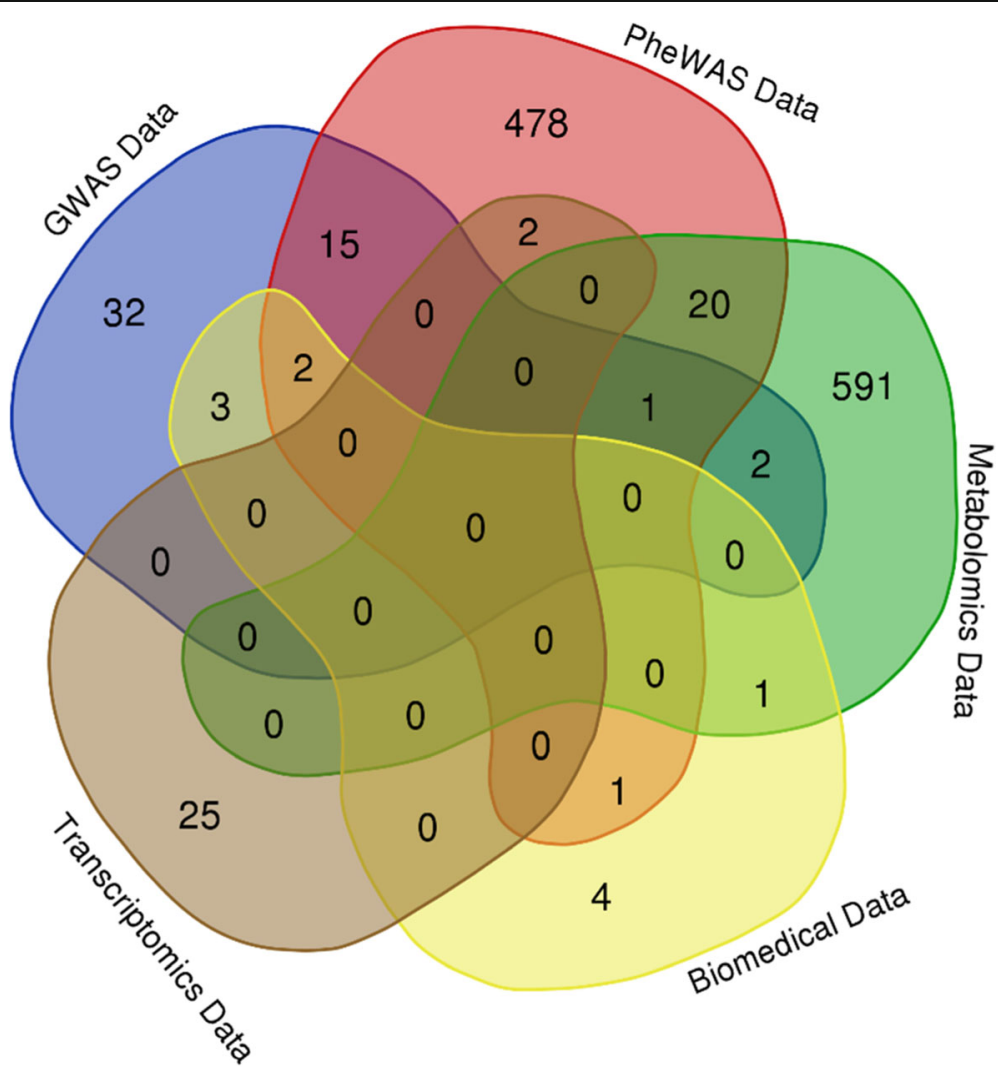

Fig. 2 Venn diagram showing the logical distribution of melanoma associated genes and proteins from revealed from various approaches. Here we have five different datasets for melanoma responsive genes or proteins. There are 50 melanoma associated genes or proteins discovered from more than one approach showing high accuracy in disease related target identification 


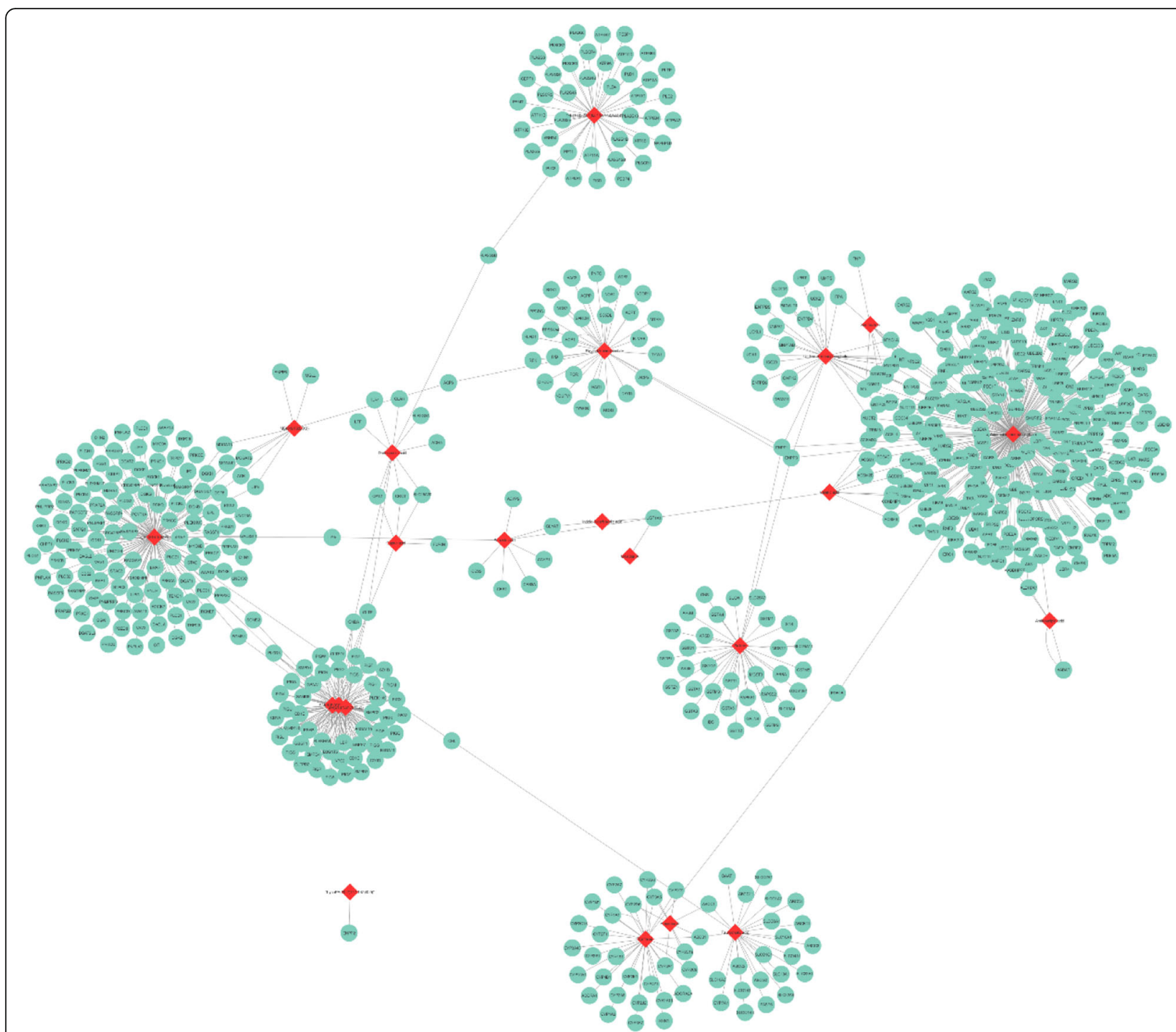

Fig. 3 Metabolites-protein network reconstruction for cutaneous malignant melanoma; red node represent metabolites and green nodes signify proteins in the form of enzymes and transporters and the edges shows their common association which has been revealed from HMDB. There are 23 unique metabolites connecting to 617 associated enzymes or transporters

(362 drugs associated with 33 targets) and carrier (6 drugs associated with 4 targets). We have mapped all types of targets with the melanoma risk biomarkers to find druggable targets. Hence, by analyzing over 1170 genes, we left with 193 mapping targets. We found 731 potential drug molecules in this step, which are available in supplementary file 3 (https://github.com/LBBSoft/Melanoma). We retrieved the drug name, transporter action, target name, indication, FDA label and DrugBank ID.

\section{Unknown melanoma potential drug}

We eliminated the drug molecules with melanoma indications from our proposed drugs. There are 215 melanomarelated drug compounds in phase 0 to 4 with different statuses, such as completed, recruited, suspended, withdrawn, not yet recruited and terminated. We found 75 common drug compounds in both melanoma-related drugs and in potential drug compounds. By eliminating common drug lists from our potential drug compound, we left with 658 drug molecules associated with 184 targets.

\section{Melanoma drug pathogenesis analysis}

We used OMIM (Amberger et al. 2015) and other scientific reports to find pathogenic information on various targets. As the pathogenesis is mainly correlated to genetic disorders, we have looked to find pathogenic information on the melanoma disorder. We considered the pathogenic information of 74 targets associated with 361 drug compounds. 
We have analyzed the effect of the pathogenic gene function (loss and gain of function) to drug-targets looking at the transporter actions (agonist and antagonist) retrieved for each candidate drugs from the DrugBank. By removing nonmatching genes, whose activities do not suit their pathogenetic information, we obtained 277 potential drugs interacting with 74 targets. The related data in this step is available in supplementary file 4 (https:// github.com/LBBSoft/Melanoma).

\section{Connectivity map analysis}

We evaluated the negative association of the "selegiline" drug, which inhibits the apoptosis in M-1 human melanoma cells and the positive association of anti-melanomarelated drugs such as "paclitaxel" and "vinblastine" to obtain a suitable expressional effect on the treatment of the melanoma disorder. We revealed the drug expression profile by looking for different drugs based on a signature query in CMap. As CMap covers the multiple drug expression in human cell lines, it will pick the most expressed drug in the available human cell line. Hence, we have achieved our final anti-melanoma potential drugs by utilizing the correlation of known anti-melanoma and proposed drugs with the help of the mean and enrichment score. Many of the drug compounds did not have a positive correlation with known anti-melanoma drugs, and some were not found in the CMap database. We left with 35 drug compounds that are associated with melanoma therapy. These drug compounds correspond to 20 targets of the protein, enzyme and transporter types. The final repurposed drug candidates are shown in Table 1.

These drugs have an enrichment score in the range of -0.167 to -0.755 . The $p$-value and specificity of most of the repurposed drug were not available. The CMap detail of the repurposed drug is available in supplementary file 5 (https://github.com/LBBSoft/Melanoma).

\section{Discussion}

In this study, we used a wide range of data analyses to find the responsive genetic content to reveal potential melanoma-related targets. These targeting genes and proteins have associated drug compounds that are approved but have not been reported as melanomatreating drugs. We found 35 drug candidates interacting with our analyzed set of targeted genes and proteins. These drugs are of different human pathogeneses and action modes, which have a treatment efficacy over melanoma patients. Apart from that, based on the analysis of the connectivity map we found, our candidate drugs have a positive association of melanoma known drugs expression profile over human cell lines.

As is shown in Table 1, we found supporting biological evidence for the applicability of 30 drug compounds as potential drug candidates for the treatment of melanoma in previous scientific reports. These reports concentrated on finding a feasible effect of drug candidates in melanoma or skin cancer cell lines or various other animal tests. There are only 5 drug candidates, which have no related previous studies showing their anti-melanoma effects.

The repurposed drug candidates are highly effective in the inhibition of cell proliferation in melanoma cells. The induction of apoptosis in melanoma cells is also one of major results of the drug's effects on the disease. There are other reports stating the effect of various drugs in the alteration of the melanoma cellular function, which can include various metabolic functions that alter the energy level of metabolites in melanoma cells and their effect on mitochondrial and other pathways.

We used the most precise target identification approaches to select melanoma-related genes and proteins. These approaches include all types of genetic analyses, including genetic variation, expression profiling, biomedical associations and metabolomics pathways. Target level analyses of given genes and proteins show that the resulting candidate drug targets are from 20 unique genes and proteins. These genes have been selected from a various wide range of data analyses. The revealed genes and protein distributions are as follows: 6 targets from PheWAS data, 3 targets from GWAS data, 14 targets from metabolomics data and 2 targets from biomedical data and no selected targets from transcriptomics data. The list of gene/protein names followed by the method of analysis is given in supplementary file 6 (https:// github.com/LBBSoft/Melanoma).

We have seen the TYR, TERT, CYP1B1, CYP19A1 and CYP1A1 genes/proteins, which are the candidate drug targets fetched by more than one method of analysis. TYR is revealed in PheWAS, GWAS and biomedical datasets. TERT is revealed in GWAS and biomedical datasets. CYP1B1 is found in the metabolomics and GWAS dataset. Finally, CYP19A1 and CYP1A1 are found in both the metabolomic and PheWAS datasets.

The connectivity map (CMap) analysis includes repurposed drugs with high positive (paclitaxel and vinblastine) or negative (selegiline) correlations of mean and enrichment scores; hence, only drugs with a similar mechanism of action will be included in this approach. However, this method may bypass some anti-melanoma potential drugs with a novel mechanism of action, which can be noted as the limitation of this approach.

This in silico approach can be used for various disorders and has a high potential for nominating sets of novel repurposed drugs with higher performance. This approach used a wide range of data analyses with the help of various datasets to invoke responsive genes and proteins. As databases do not cover only curated data and contains predictive and putative data, databases may contain several false-positive or false-negative data. 


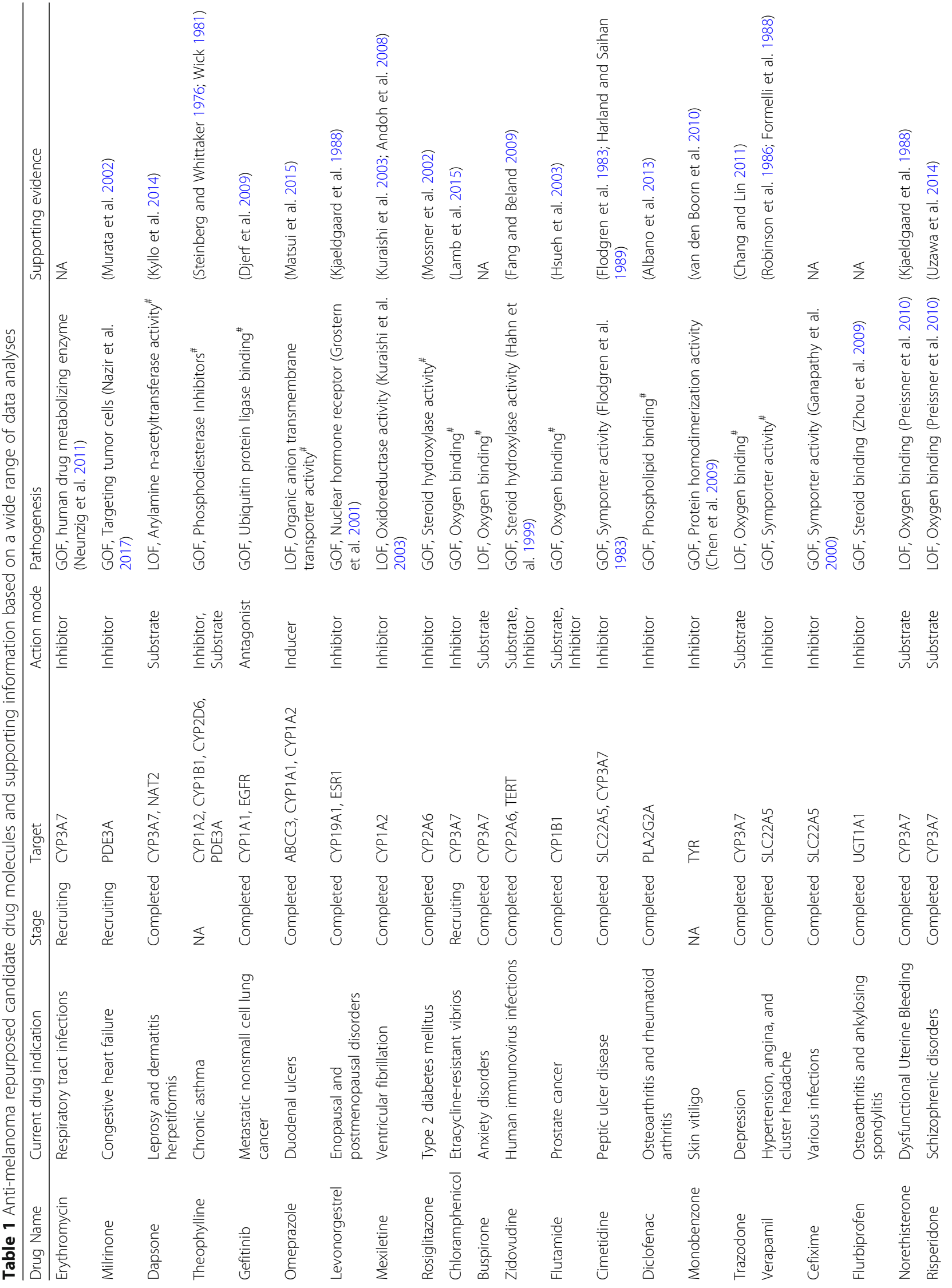




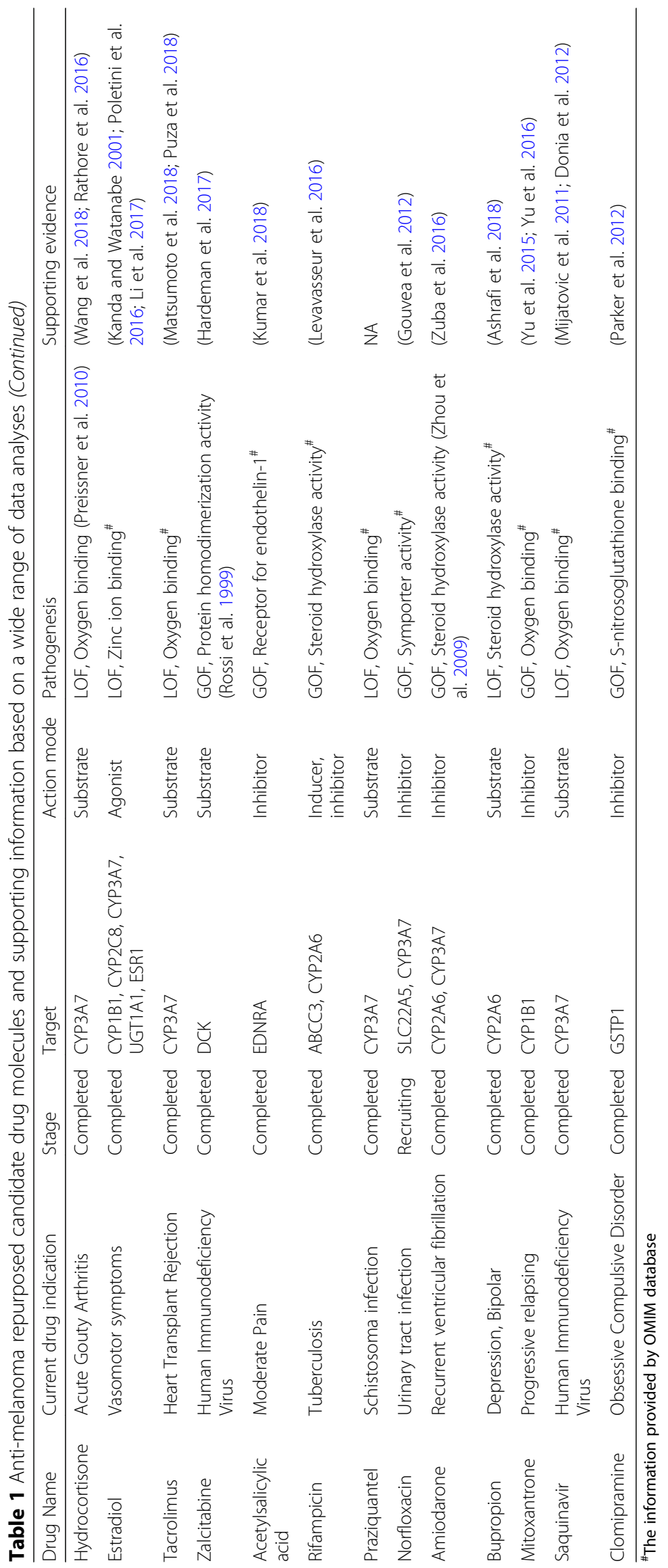


Apart from that, an analysis of CMap did not consider the expression of drugs on melanoma-specific cell lines and may have few errors. Therefore, in vitro or in vivo experiments and, later, animal and clinical trials are required to repurpose these candidate drugs.

\section{Conclusions}

Cutaneous malignant melanoma is a highly dangerous disorder that has high mortality and less survival time. Due to the non-availability of treatment drugs, treating the disease is costly and painful. We have used a wide range of analyses to reveal the melanoma-related influencing genes and found corresponding druggable proteins. In this methodology, we excluded known melanoma drugs and their respected targets from the dataset. The pathogenesis information of selected targets has been analyzed based on the disorder and pharmaceutical actions. Furthermore, the resulting drug targets have been analyzed based on the expression profile of drugs to the human cell line with the help of the CMap Tool. We found 35 potential drugs interacting with 20 targets, which can treat melanoma disorder. This approach can be used to find potential treatment drugs for other disorders.

\begin{abstract}
Abbreviations
CMap: Connectivity map; FDA: Food and Drug Administration; GOF: Gain of function; GWAS: Genome Wide Association Studies; HMDB: Human Metabolome Database; LOF: Loss of function; OMIM: Online Mendelian Inheritance in man; PheWAS: Phenome Wide Association Studies; SNP: Single Nucleotide Polymorphism; UniProt: Universal Protein resource; UV: Ultraviolet
\end{abstract}

\section{Acknowledgments}

The authors thank Laboratory of Systems Biology \& Bioinformatics (LBB) of Institute of Biochemistry and Biophysics (IBB) of University of Tehran. We are grateful to Mr. Yousef Masoudi Sobhanzadeh and Mr. Morteza Kouhsar for their valuable technical comments.

\section{Authors' contributions}

AK conceived and designed the experiments, analyzed the data, and wrote the manuscript; $A M, B G$ and $B J$ revised the manuscript and designed the experiments; All authors read and approved the final manuscript.

\section{Funding}

Funding from the Institute of Biochemistry and Biophysics, University of Tehran is gratefully acknowledged.

\section{Availability of data and materials}

The datasets generated and/or analyzed during the current study are available from the corresponding author upon reasonable request.

\section{Ethics approval and consent to participate}

Not applicable.

\section{Consent for publication}

Not applicable.

\section{Competing interests}

The authors declare that they have no competing interests.

\section{Author details}

'Laboratory of Systems Biology and Bioinformatics (LBB), Institute of Biochemistry and Biophysics, University of Tehran, Tehran, Iran. ${ }^{2}$ Department of Chemistry \& Supercomputing Facility for Bioinformatics \& Computational
Biology, Kusuma School of Biological Sciences, Indian Institute of Technology, Delhi, India. ${ }^{3}$ Department of Biophysics \& Bioinformatics, Institute of Biochemistry and Biophysics (IBB), University of Tehran, Tehran, Iran.

Received: 3 February 2019 Accepted: 5 June 2019

Published online: 20 June 2019

\section{References}

Albano F, Arcucci A, Granato G, Romano S, Montagnani S, De Vendittis E, et al. Markers of mitochondrial dysfunction during the diclofenac-induced apoptosis in melanoma cell lines. Biochimie. 2013;95:934-45.

Amberger JS, Bocchini CA, Schiettecatte F, Scott AF, Hamosh A. OMIM.org: online Mendelian inheritance in man $(\mathrm{OMIM}(\mathrm{R}))$, an online catalog of human genes and genetic disorders. Nucleic Acids Res. 2015;43:D789-98.

Andoh T, Sugiyama K, Fujita M, lida Y, Nojima H, Saiki I, et al. Pharmacological evaluation of morphine and non-opioid analgesic adjuvants in a mouse model of skin cancer pain. Biol Pharm Bull. 2008;31:520-2.

Ashburn TT, Thor KB. Drug repositioning: identifying and developing new uses for existing drugs. Nat Rev Drug Discov. 2004;3:673-83.

Ashrafi F, Mousavi S, Karimi M. Potential role of bupropion sustained release for cancer-related fatigue: a double-blind, placebo-controlled study. Asian Pac J Cancer Prev. 2018;19:1547-51.

Atak ZK, Gianfelici V, Hulselmans G, De Keersmaecker K, Devasia AG, Geerdens E, et al. Comprehensive analysis of transcriptome variation uncovers known and novel driver events in T-cell acute lymphoblastic leukemia. PLoS Genet. 2013;9:e1003997.

Babbi G, Martelli PL, Profiti G, Bovo S, Savojardo C, Casadio R. eDGAR: a database of disease-gene associations with annotated relationships among genes. BMC Genomics. 2017;18:554.

Balch CM, Gershenwald JE, Soong SJ, Thompson JF, Atkins MB, Byrd DR, et al. Final version of 2009 AJCC melanoma staging and classification. J Clin Oncol Off J Am Soc Clin Oncol. 2009;27:6199-206.

Berger MF, Levin JZ, Vijayendran K, Sivachenko A, Adiconis X, Maguire J, et al. Integrative analysis of the melanoma transcriptome. Genome Res. 2010;20:413-27.

Bush WS, Oetjens MT, Crawford DC. Unravelling the human genome-phenome relationship using phenome-wide association studies. Nat Rev Genet. 2016; $17: 129-45$

Chang TS, Lin VC. Melanogenesis inhibitory activity of two generic drugs: cinnarizine and trazodone in mouse B16 melanoma cells. Int J Mol Sci. 2011; 12:8787-96.

Chen YR, Y-Y R, Lin TY, Huang CP, Tang WC, Chen ST, et al. Identification of an Alkylhydroquinone from Rhus succedanea as an inhibitor of Tyrosinase and Melanogenesis. J Agric Food Chem. 2009;57:2200-5.

Cummins DL, Cummins JM, Pantle H, Silverman MA, Leonard AL, Chanmugam A. Cutaneous malignant melanoma. Mayo Clin Proc. 2006;81:500-7.

Denny JC, Bastarache L, Ritchie MD, Carroll RJ, Zink R, Mosley JD, et al. Systematic comparison of phenome-wide association study of electronic medical record data and genome-wide association study data. Nat Biotechnol. 2013;31: 1102-11.

Djerf EA, Trinks C, Abdiu A, Thunell LK, Hallbeck AL, Walz TM. ErbB receptor tyrosine kinases contribute to proliferation of malignant melanoma cells: inhibition by gefitinib (ZD1839). Melanoma Res. 2009;19:156-66.

Donia M, Mangano K, Fagone P, De Pasquale R, Dinotta F, Coco M, et al. Unique antineoplastic profile of Saquinavir-NO, a novel NO-derivative of the protease inhibitor Saquinavir, on the in vitro and in vivo tumor formation of A375 human melanoma cells. Oncol Rep. 2012;28:682-8.

Elder D. Tumor progression, early diagnosis and prognosis of melanoma. Acta Oncol. 1999:38:535-48.

Fang $J$, Beland FA. Long-term exposure to zidovudine delays cell cycle progression, induces apoptosis, and decreases telomerase activity in human hepatocytes. Toxicol Sci. 2009;111:120-30.

Flodgren P, Borgstrom S, Jonsson PE, Lindstrom C, Sjogren HO. Metastatic malignant melanoma: regression induced by combined treatment with interferon [HulFN-alpha(Le)] and cimetidine. Int J Cancer. 1983;32:657-65.

Formelli F, Supino R, Cleris L, Mariani M. Verapamil potentiation of doxorubicin resistance development in B16 melanoma cells both in vitro and in vivo. Br J Cancer. 1988:57:343-7.

Ganapathy ME, Huang W, Rajan DP, Carter AL, Sugawara M, Iseki K, et al. Betalactam antibiotics as substrates for OCTN2, an organic cation/carnitine transporter. J Biol Chem. 2000;275:1699-707. 
Gouvea LR, Garcia LS, Lachter DR, Nunes PR, de Castro Pereira F, Silveira-Lacerda $E P$, et al. Atypical fluoroquinolone gold(III) chelates as potential anticancer agents: relevance of DNA and protein interactions for their mechanism of action. Eur J Med Chem. 2012:55:67-73.

Grostern RJ, Slusker Shternfeld I, Bacus SS, Gilchrist K, Zimbric ML, Albert DM. Absence of type I estrogen receptors in choroidal melanoma: analysis of collaborative ocular melanoma study (COMS) eyes. Am J Ophthalmol. 2001;131:788-91.

Hahn WC, Counter CM, Lundberg AS, Beijersbergen RL, Brooks MW, Weinberg RA. Creation of human tumour cells with defined genetic elements. Nature. 1999:400:464-8.

Han J, Colditz GA, Liu JS, Hunter DJ. Genetic variation in XPD, sun exposure, and risk of skin cancer. Cancer Epidemiol Prev Biomark. 2005;14:1539-44.

Hardeman KN, Peng C, Paudel BB, Meyer CT, Luong T, Tyson DR, et al. Dependence on glycolysis sensitizes BRAF-mutated melanomas for increased response to targeted BRAF inhibition. Sci Rep. 2017;7:42604.

Harland CC, Saihan EM. Regression of cutaneous metastatic malignant melanoma with topical diphencyprone and oral cimetidine. Lancet (London, England). 1989;2:445.

Hsueh EC, Gupta RK, Lefor A, Reyzin G, Ye W, Morton DL. Androgen blockade enhances response to melanoma vaccine. J Surg Res. 2003;110:393-8.

Kanda N, Watanabe S. 17beta-estradiol, progesterone, and dihydrotestosterone suppress the growth of human melanoma by inhibiting interleukin-8 production. J Invest Dermatol. 2001;117:274-83.

Khosravi A, Kouhsar M, Goliaei B, Jayaram B, Masoudi-Nejad A. Systematic analysis of genes and diseases using PheWAS-associated networks. Comput Biol Med. 2019;109:311-21.

Kjaeldgaard A, Ahlesteen I, Larsson B, Astedt B. Progestogen regulation of tissue plasminogen activator in a human melanoma cell line. Thromb Res. 1988;49:287-97.

Kumar D, Rahman H, Tyagi E, Liu T, Li C, Lu R, et al. Aspirin suppresses PGE2 and activates AMP kinase to inhibit melanoma cell motility, pigmentation, and selective tumor growth in vivo. Cancer Prev Res (Phila). 2018;11:629-42.

Kumar-Sinha C, Kalyana-Sundaram S, Chinnaiyan AM. Landscape of gene fusions in epithelial cancers: seq and ye shall find. Genome Med. 2015;7:129.

Kuraishi Y, lida Y, Zhang HW, Uehara S, Nojima H, Murata J, et al. Suppression by gabapentin of pain-related mechano-responses in mice given orthotopic tumor inoculation. Biol Pharm Bull. 2003:26:550-2.

Kyllo RL, Parker MK, Rosman I, Musiek AC. Ipilimumab-associated sweet syndrome in a patient with high-risk melanoma. J Am Acad Dermatol. 2014;70:e85-6.

Lamb J, Crawford ED, Peck D, Modell JW, Blat IC, Wrobel MJ, et al. The connectivity map: using gene-expression signatures to connect small molecules, genes, and disease. Science. 2006;313:1929-35.

Lamb R, Ozsvari B, Lisanti CL, Tanowitz HB, Howell A, Martinez-Outschoorn UE, et al. Antibiotics that target mitochondria effectively eradicate cancer stem cells, across multiple tumor types: treating cancer like an infectious disease. Oncotarget. 2015;6:4569-84.

Landrum MJ, Lee JM, Benson M, Brown G, Chao C, Chitipiralla S, et al. ClinVar: public archive of interpretations of clinically relevant variants. Nucleic Acids Res. 2016;44:D862-8.

Levavasseur M, Darras S, Mortier L, Goeminne C, Auffret M, Bertrand M. Drug interaction between dabrafenib and immunosuppressive drugs: about one case. Melanoma Res. 2016;26:532-4.

Li J, Zheng S, Chen B, Butte AJ, Swamidass SJ, Lu Z. A survey of current trends in computational drug repositioning. Brief Bioinform. 2016;17:2-12.

Li L, Feng J, Chen Y, Li S, Ou M, Sun W, et al. Estradiol shows anti-skin cancer activities through decreasing MDM2 expression. Oncotarget. 2017:8:8459-74.

MacArthur J, Bowler E, Cerezo M, Gil L, Hall P, Hastings E, et al. The new NHGRI$\mathrm{EB}$ l catalog of published genome-wide association studies (GWAS catalog). Nucleic Acids Res. 2017;45:D896-901

Matsui MS, Petris MJ, Niki Y, Karaman-Jurukovska N, Muizzuddin N, Ichihashi M, et al. Omeprazole, a gastric proton pump inhibitor, inhibits melanogenesis by blocking ATP7A trafficking. J Invest Dermatol. 2015;135:834-41.

Matsumoto H, Sato S, Fujita Y, Yashiro-Furuya M, Matsuoka N, Asano T, et al. Rheumatoid arthritis complicated with anti-melanoma differentiationassociated gene 5 antibody-positive interstitial pneumonia: a case report. Intern Med. 2018;58(5):737-42.

Mijatovic S, Maksimovic-Ivanic D, Mojic M, Timotijevic G, Miljkovic D, Mangano K, et al. Cytotoxic and immune-sensitizing properties of nitric oxide-modified Saquinavir in iNOS-positive human melanoma cells. J Cell Physiol. 2011;226:1803-12.

Mossner R, Schulz U, Kruger U, Middel P, Schinner S, Fuzesi L, et al. Agonists of peroxisome proliferator-activated receptor gamma inhibit cell growth in malignant melanoma. J Invest Dermatol. 2002;119:576-82.
Murata T, Shimizu K, Narita M, Manganiello VC, Tagawa T. Characterization of phosphodiesterase 3 in human malignant melanoma cell line. Anticancer Res. 2002;22:3171-4.

Nazir M, Senkowski W, Nyberg F, Blom K, Edqvist PH, Jarvius M, et al. Targeting tumor cells based on phosphodiesterase 3A expression. Exp Cell Res. 2017;361:308-15.

Neunzig I, Dragan CA, Widjaja M, Schwaninger AE, Peters FT, Maurer HH, et al. Whole-cell biotransformation assay for investigation of the human drug metabolizing enzyme CYP3A7. Biochim Biophys Acta. 2011;1814:161-7.

UniProt Consortium. UniProt: a hub for protein information. Nucleic Acids Res. 2015;43:D204-12

Parker KA, Glaysher S, Hurren J, Knight LA, McCormick D, Suovouri A, et al. The effect of tricyclic antidepressants on cutaneous melanoma cell lines and primary cell cultures. Anti-Cancer Drugs. 2012;23:65-9.

Parkin DM, Mesher D, Sasieni P. 13. Cancers attributable to solar (ultraviolet) radiation exposure in the UK in 2010. Br J Cancer. 2011;105(Suppl 2):S66-9.

Poletini MO, de Assis LV, Moraes MN, Castrucci AM. Estradiol differently affects melanin synthesis of malignant and normal melanocytes: a relationship with clock and clock-controlled genes. Mol Cell Biochem. 2016;421:29-39.

Preissner S, Kroll K, Dunkel M, Senger C, Goldsobel G, Kuzman D, et al. SuperCYP: a comprehensive database on cytochrome P450 enzymes including a tool for analysis of CYP-drug interactions. Nucleic Acids Res. 2010;38:D237-43.

Puza CJ, Cardones AR, Mosca PJ. Examining the incidence and presentation of melanoma in the cardiothoracic transplant population. JAMA Dermatol. 2018; 154:589-91.

Rastegar-Mojarad M, Ye Z, Kolesar JM, Hebbring SJ, Lin SM. Opportunities for drug repositioning from phenome-wide association studies. Nat Biotechnol. 2015;33:342-5.

Rathore B, Chandra Sekhar MM, Jaggarapu A, Ganguly HK, Reddy Rachamalla RB. Cationic lipid-conjugated hydrocortisone as selective antitumor agent. Eur J Med Chem. 2016;108:309-21.

Robertson DG, Frevert U. Metabolomics in drug discovery and development. Clin Pharmacol Ther. 2013:94:559-61.

Robinson BA, Clutterbuck RD, Millar JL, McElwain TJ. Effects of verapamil and alcohol on blood flow, melphalan uptake and cytotoxicity, in murine fibrosarcomas and human melanoma xenografts. Br J Cancer. 1986:53:607-14

Rossi L, Serafini S, Schiavano GF, Casabianca A, Vallanti G, Chiarantini L, et al. Metabolism, mitochondrial uptake and toxicity of 2', 3'-dideoxycytidine. Biochem J. 1999;344(Pt 3):915-20.

Shannon P, Markiel A, Ozier O, Baliga NS, Wang JT, Ramage D, et al. Cytoscape: a software environment for integrated models of biomolecular interaction networks. Genome Res. 2003;13:2498-504.

Srivastava A, Creek DJ. Discovery and validation of clinical biomarkers of cancer: a review combining metabolomics and proteomics. Proteomics. 2018;19: e1700448.

Steinberg ML, Whittaker JR. Stimulation of melanotic expression in a melanoma cell line by theophylline. J Cell Physiol. 1976;87:265-75.

Sun P, Guo J, Winnenburg R, Baumbach J. Drug repurposing by integrated literature mining and drug-gene-disease triangulation. Drug Discov Today. 2017;22:615-9.

Uzawa K, Kasamatsu A, Shimizu T, Saito Y, Baba T, Sakuma K, et al. Suppression of metastasis by mirtazapine via restoration of the Lin-7C/beta-catenin pathway in human cancer cells. Sci Rep. 2014:4:5433.

van den Boorn JG, Konijnenberg D, Tjin EP, Picavet DI, Meeuwenoord NJ, Filippov $\mathrm{DV}$, et al. Effective melanoma immunotherapy in mice by the skindepigmenting agent monobenzone and the adjuvants imiquimod and CpG. PLoS One. 2010;5:e10626.

Wang F, Li B, Fu P, Li Q, Zheng H, Lao X. Immunomodulatory and enhanced antitumor activity of a modified thymosin alpha1 in melanoma and lung cancer. Int J Pharm. 2018:547:611-20.

Wang Z-Y, Zhang H-Y. Rational drug repositioning by medical genetics. Nat Biotechnol. 2013;31:1080.

Wick MM. Inhibition of clonogenic growth of melanoma cells by combination of melanocyte stimulating hormone and theophylline. J Invest Dermatol. 1981; 77:253-5.

Wishart DS, Feunang YD, Guo AC, Lo EJ, Marcu A, Grant JR, et al. DrugBank 5.0: a major update to the DrugBank database for 2018. Nucleic Acids Res. 2018b; 46:D1074-d1082

Wishart DS, Feunang YD, Marcu A, Guo AC, Liang K, Vazquez-Fresno R, et al. HMDB 4.0: the human metabolome database for 2018. Nucleic Acids Res. 2018a;46:D608-d617. 
Yin W, Gao C, Xu Y, Li B, Ruderfer DM, Chen Y. Learning opportunities for drug repositioning via GWAS and PheWAS findings, AMIA joint summits on translational science proceedings. AMIA Joint Summits Transl Sci. 2018;2017: 237-46.

Yu X, Du L, Li Y, Fu G, Jin Y. Improved anti-melanoma effect of a transdermal mitoxantrone ethosome gel. Biomed Pharmacother. 2015;73:6-11.

Yu X, Du L, Zhu L, Liu X, Zhang B, Fu G, et al. Melanoma therapy with transdermal mitoxantrone cubic phases. Drug Deliv. 2016;23:1565-70.

Zhang M, Luo H, Xi Z, Rogaeva E. Drug repositioning for diabetes based on 'Omics' data mining. PLoS One. 2015;10(5):e0126082.

Zhao S, Fung-Leung WP, Bittner A, Ngo K, Liu X. Comparison of RNA-Seq and microarray in transcriptome profiling of activated T cells. PLoS One. 2014;9: e78644.

Zhou SF, Zhou ZW, Yang LP, Cai JP. Substrates, inducers, inhibitors and structureactivity relationships of human cytochrome P450 2C9 and implications in drug development. Curr Med Chem. 2009;16:3480-675.

Zuba EB, Francuzik W, Malicki P, Osmola-Mankowska A, Jenerowicz D. Knowledge about ultraviolet radiation hazards and tanning behavior of cosmetology and medical students. Acta Dermatovenerol Croat. 2016;24:73-7.

\section{Publisher's Note}

Springer Nature remains neutral with regard to jurisdictional claims in published maps and institutional affiliations.

Ready to submit your research? Choose BMC and benefit from:

- fast, convenient online submission

- thorough peer review by experienced researchers in your field

- rapid publication on acceptance

- support for research data, including large and complex data types

- gold Open Access which fosters wider collaboration and increased citations

- maximum visibility for your research: over $100 \mathrm{M}$ website views per year

At $\mathrm{BMC}$, research is always in progress.

Learn more biomedcentral.com/submissions 